\title{
TEM Study of Pt Cluster Incorporated Zeolite A
}

\author{
Y. Shi, ${ }^{*}$ F. Wang, $* *$ J. Qian, ${ }^{* *}$ H. Yang, ${ }^{*}$ L. Ding, ${ }^{*}$ P. Li, ${ }^{* *}$ S. Ng, ${ }^{*}$ J. Chen, ${ }^{*}$ M. Malac** \\ * National Centre for Upgrading Technology (NCUT), CanmetENERGY,Natural Resources Canada, \\ Devon, Alberta, T9G 1 A8 \\ ** National Institute for Nanotechnology (NINT), 11421 Saskatchewan Drive, Edmonton, T6G 2M9
}

Zeolite supported noble metal catalysts provide simultaneously both hydrogenation active sites and acid sites for hydrocracking reaction, which have been widely used in petroleum hydroprocessing processes. The size, location and distribution of the metal particles, as well as the pore structure and crystallinity of the zeolite support are the key factors affecting the activity and selectivity of the catalyst. To enhance the activity and durability of the catalyst, it is of great importance to obtain highly dispersed noble metal clusters within zeolite support. It is of particular importance to introduce small metal particles into the confines of the zeolite micropores. While the conventional ion exchange method works only for inserting metal ion precursors into larger pore zeolite such as zeolite Y, it is difficult to add metal precursors into the cavity of zeolite A due to the small pore size of $\mathrm{NaA}$.

So far, much effort has been devoted to introducing Pt into zeolite A by adding a platinum precursor to the synthesis mixture containing both Al and Si source [1,2]. Although preliminary results of chemisorption and shape-selective catalytic test have indicated that the platinum clusters located inside the larger cavity ( $\alpha$ cage) of zeolite A [1,3-5], the direct information on the location and distribution of Pt particles, and the microstructure of zeolite support after loading is still in shortage. Transmission electron microscopy (TEM) can provide insight into the structure (atomic/nanoscopic), crystallography and chemical composition of solid catalysts. Our SEM and Xray diffraction (XRD) study has shown that both morphology and crystallinity of the synthesized zeolite changed when a platinum precursor was incorporated into the initial synthesis mixture (see Fig. 1) [5]. Here, we present the experimental results of the Pt nanoparticles and the microstructure of the aforementioned samples in TEM (JEOL 2200FS). To obtain ultrathin sections ( 40 nm) of the zeolite catalysts, samples were first embedded in epoxy resin, and then microtomed and collected onto a carbon coated $\mathrm{Cu}$-grid.

Figure 2 presents the high angle annular dark field (HAADF) STEM image for the high Pt loaded sample, showing that very small (less than $2 \mathrm{~nm}$ ) Pt particles are evenly distributed within the support. The diffraction rings can be attributed to Face Centered Cubic (FCC) platinum, and no obvious reflections from zeolite were observed. Figure 3 shows the STEM image taken from 0.69 wt $\%$ Pt loaded sample. Selected area electron diffraction (SAED) patterns recorded from different particles indicate that the large particles ( $\mu \mathrm{m}$ scale in Fig. 1) are crystalline while the smaller ones with rough surfaces (see inset of Fig. 1b) are amorphous. The sharp diffraction rings originate from Pt. Electron Energy Loss Spectra (EELS) were also taken for local structural and chemical analysis. Figure 4 shows a comparison of EELS spectra of O-K edge between NaA and high Pt loaded sample after background subtraction and Fourier-ratio deconvolution [6]. The dramatic change in O-K edge, as a consequence of local environmental change with Pt loading, indicates the breakdown of the cage structure. We believe that the pre-peak does not originate from free $\mathrm{O}_{2}$ molecules, because the intensity relative to the major peak doesn't change with dose and dose rate [7]. Presumably it arises from the direct bonding of oxygen with sodium after the cage breaks. The changes of the near-edge structures of the $\mathrm{O}-\mathrm{K}$ are being further investigated by multiple scattering calculations and 
controlled experiments.

References

[1] R. Vijayalakshmi et al., Solid State Sci. 4 (2002) 489.

[2] P.B. Weisz et al., J. Catal. 1 (1962) 307.

[3] A. Joseph et al., J. Chem. Soc. Chem. Commun. (1986) 234.

[4] H. Yang et al., J. Catal. 243 (2006) 36-42.

[5] H. Yang et al., Micropor. Mesopor. Mat. 117 (2009) 33.

[6] R.F. Egerton, Electron Energy-Loss Spectroscopy in the Electron Microscope, Plenum/Kluwer /Springer, New York (1996).

[7] N. Jiang et al., Ultramicroscopy 106 (2006) 215.

[8] This research was supported by Canadian Program for Energy Research and Development, Alberta Research Council, Alberta Energy Research Institute, NCUT and NINT.
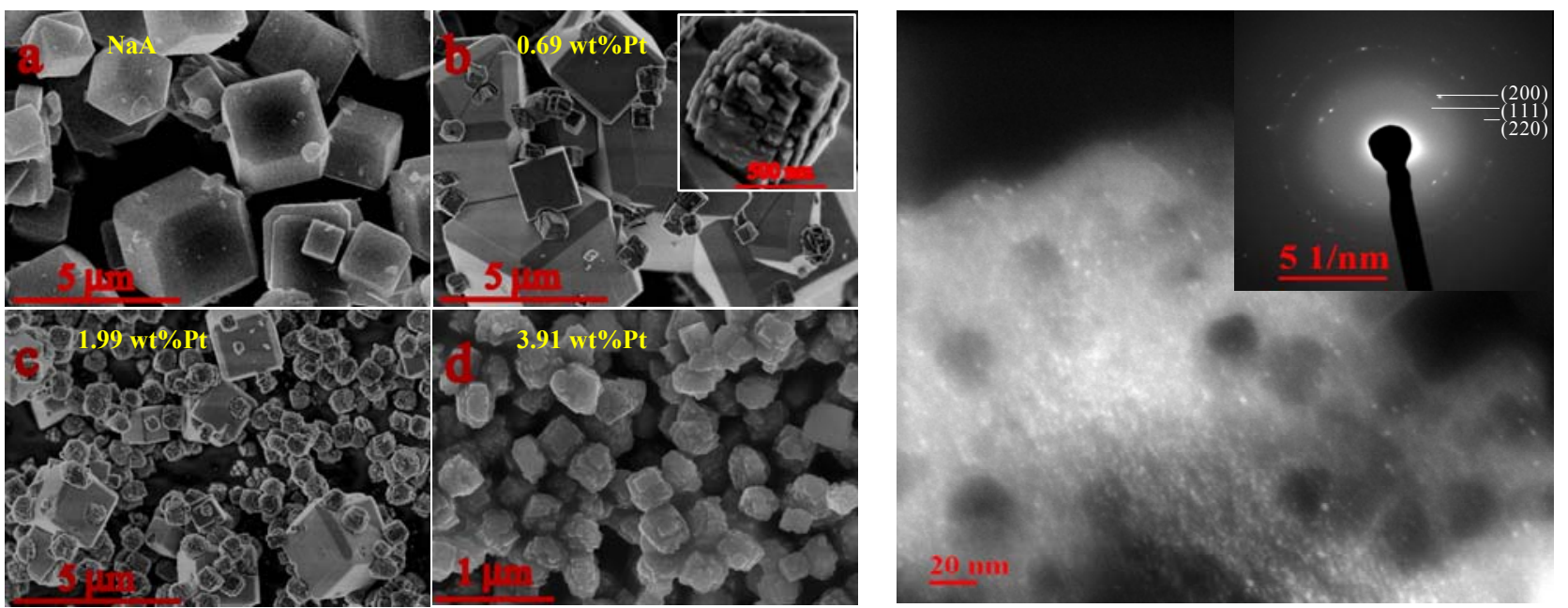

FIG. 1. SEM images of the catalysts with different Pt loading content.

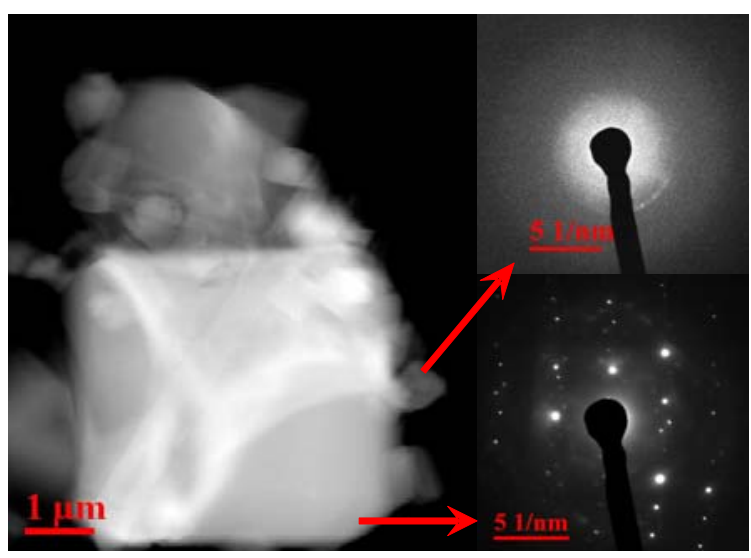

FIG. 3. STEM image of $0.5 \mathrm{wt} \% \mathrm{Pt}$ loaded sample and the corresponding SAED patterns taken from different particles.
FIG. 2. HAADF image of $4 \mathrm{wt} \% \mathrm{Pt}$ loaded sample.

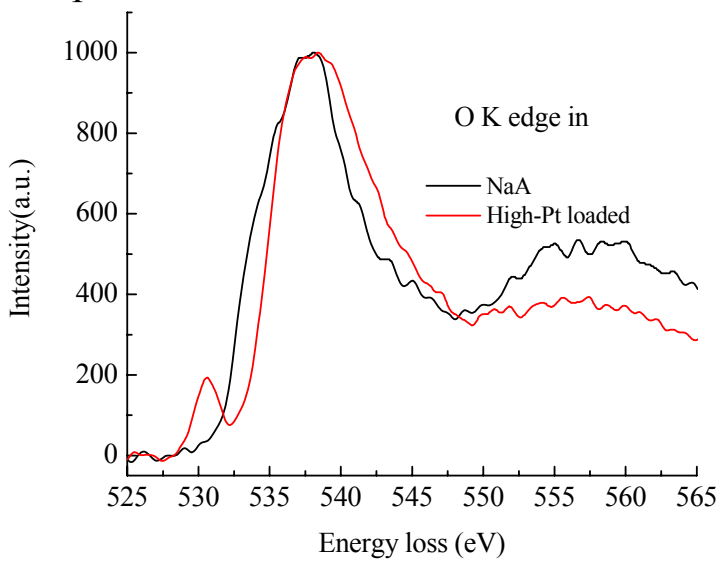

FIG. 4. EEL spectra of O K-edge. 